\title{
Sleeping posture and intraocular pressure
}

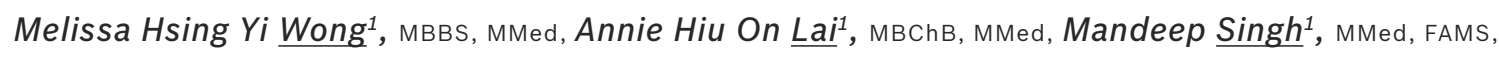
Paul Tec Kuan Chew ${ }^{1}$, MMed, FAMs

INTRODUCTION This prospective observational case series aimed to determine whether the lateral decubitus position, which is commonly adopted during sleep, has an effect on intraocular pressure (IOP) in normal controls.

METHODS Patients without glaucoma were recruited from those visiting outpatient clinics for non-glaucomatous conditions. The left eye of each patient was included. IOP was first measured using Tono-Pen ${ }^{\circledR} \mathrm{XL}$ applanation tonometer in the supine position, following which a second measurement was immediately obtained for the left lateral head position. Measurements were obtained with the patient lying on one soft and one hard pillow for each position, and patients remained awake during these measurements. One tonometry reading was obtained for each position. Readings were recorded only when the average of four independent readings produced a statistical confidence index of $5 \%$. Results were analysed using the paired Student's $t$-test for comparison of the means.

RESULTS IOP in the left lateral decubitus position $(17.48 \pm 3.18 \mathrm{mmHg})$ was significantly higher than in the supine position $(14.48 \pm 3.09 \mathrm{mmHg})$ when using soft pillows $(p<0.001)$. When hard pillows were used, IOP in the left lateral decubitus position also exceeded that measured in the supine position $(16.65 \pm 3.54 \mathrm{mmHg}$ vs. $13.65 \pm 3.58 \mathrm{mmHg}$; $p<0.001)$. There was no statistically significant difference in the IOPs measured for the same position when different kinds of pillows were used.

CONCLUSION The lateral decubitus position adopted during sleep is associated with changes in IOP in healthy volunteers.

Keywords: intraocular pressure, sleeping posture

\section{INTRODUCTION}

Intraocular pressure (IOP) is a measure of the fluid inside the eye. Controlling IOP has been the primary focus of glaucoma treatment. ${ }^{(1)}$ IOP is affected by various factors such as diurnal variation and posture. ${ }^{(2-6)}$ Some authors have suggested that nocturnal IOP is higher than in the waking periods. ${ }^{(2)}$ Several studies have compared IOPs in the supine and sitting positions, and found that IOP in the supine position is consistently higher than that in the sitting position. . $^{(2,4,7)}$ As such, it is believed that optimal control of IOP in glaucoma patients during sleep may be more crucial than that during the daytime. ${ }^{(4)}$ However, to date and to our knowledge, only a few studies have explored the effects of various sleeping positions such as the lateral decubitus and supine positions on IOP.(5,6)

In this study, the authors aimed to determine whether lying in a lateral decubitus position, which is a common position adopted during sleep, would contribute to an elevation in the IOP of the dependent eye as compared to lying in a supine position.

\section{METHODS}

In this prospective observational case series, informed consent was obtained from all patients. Approval for the study, which was carried out in accordance with the tenets of the Declaration of Helsinki, was obtained from the institutional ethics committee. For ease of recruitment, adult patients who were above the age of legal consent (21 years) were recruited from a general ophthalmology outpatient clinic. These patients were reviewed for conditions such as floaters and cataracts. The following patients were excluded: (a) patients who did not consent to the study or were unable to lie supine or decubitus for the study; and (b) patients with significant anterior segment pathologies (significant cataracts with visual acuity worse than 6/18 or nuclear sclerosis $3+$ or more) or posterior segment pathologies that could act as secondary causes of raised IOP.

IOP measurements were taken in a separate room at the clinic using the Tono-Pen ${ }^{\circledR} \mathrm{XL}$ applanation tonometer (Reichert Inc, Depew, NY, USA). The Tono-Pen XL was chosen, as it was more convenient to obtain IOP from patients in the lying down position using this instrument. In addition, the two observers involved in the study were familiar with its usage.

Tonometry measurements were obtained for the supine and lateral decubitus positions on two different kinds of pillows (Siong Bee Industry Pte Ltd, Singapore). Readings were recorded only if the average of four independent readings produced a statistical confidence index of $5 \%$ for each position. Patients were first asked to lie down in the supine position with the head placed on a soft pillow (moulded cold cure latex foam pillow; weight $1.1 \mathrm{~kg}$ ), and the IOP was measured within one minute of the initiation of the posture. After five minutes, the patient was instructed to lie in the left lateral decubitus position such that the left eye was dependent and in contact with the pillow surface. Care was taken to ensure that the patient's naso-occipital plane was at $45^{\circ}$ to the horizontal plane and that the pillow was at least in partial contact with the eyelids over the temporal globe. IOP was measured

${ }^{1}$ Department of Ophthalmology, National University Health System, Singapore

Correspondence: A/Prof Paul TK Chew, Senior Consultant, Department of Ophthalmology, National University Health System, 5 Lower Kent Ridge Road, Singapore 119074.ophchewp@nus.edu.sg 
again within one minute of the patient assuming this posture. The procedures to measure IOPs in the supine and left lateral decubitus positions were repeated with the patient's head placed on a hard pillow (moulded cold cure latex foam pillow; weight $1.5 \mathrm{~kg}$ ). The same pillows were used for all patients.

IOP measurements were taken between 8 am and $6 \mathrm{pm}$ for all enrolled patients (the time of measurement was not standardised). Patients remained awake during IOP estimation. All IOP estimations were performed by one of the two observers. Each observer was responsible for measuring the IOPs at the different positions using both the pillows for each patient recruited. All measurements were made using the same tonometer, and the average of four readings of IOP, each producing a statistical confidence index of $5 \%$, were taken. The readings were obtained from the left eye of all patients during the study to further standardise the measurements by each observer. The paired Student's $t$-test was used to compare the means of the IOPs obtained, and results were considered to be statistically significant at $\mathrm{p}<0.05$.

\section{RESULTS}

A total of 23 left eyes of 23 patients were included in the study. Table I shows the demographical data and the clinical diagnoses of the cohort. The majority of the patients were male $(n=15$, $65.2 \%$ ) and the mean age of the patients was $52.3 \pm 16.2$ years. Ocular diagnoses in the group included cataracts $(30.4 \%)$, dry eyes $(26.0 \%)$, blepharitis $(22.7 \%)$, vitreous syneresis $(13.0 \%)$ and others $(8.7 \%)$. IOP in the left lateral decubitus position was significantly higher than in the supine position (mean difference $-3.00 \pm 3.36 \mathrm{mmHg} ; \mathrm{p}<0.001$ ) when soft pillows were used. This difference in IOPs between the two postures was similarly significant when hard pillows were used (mean difference -3.00 $\pm 2.54 \mathrm{mmHg} ; \mathrm{p}<0.001$ ) (Tables II and III). The difference in the IOPs measured for the same position when soft and hard pillows were used was, however, not statistically significant.

\section{DISCUSSION}

We found that when a left lateral decubitus position was adopted, IOP increased in patients using both soft and hard pillows. There was a significant increase of $3 \mathrm{mmHg}$ in IOP on lying laterally compared to when lying supine. While this increase might have been, in part, a result of posture change, there is the possibility that direct mechanical compression of the pillow on the eye as the patient lay laterally plays a role in the changes in IOP. Our results bespeak the possible implications of such compression in patients with prominent facial soft tissue or exophthalmos, and whether such patients would experience higher IOP than patients with thin, bony facial structures. This is an interesting notion that could be further explored in future studies. Notably, there was no significant difference in the IOPs measured for a particular position regardless of the type of pillow used by the patients.

Korenfeld and Dueker ${ }^{(6)}$ reported a significant elevation of IOP in the dependent eye during sleep, showing a mean peak IOP of
Table I. Patient demographics and clinical diagnoses $(n=23)$.

\begin{tabular}{lr}
\hline Demographic & No. (\%) \\
\hline Mean age 士 SD (yrs) & $52.3 \pm 16.2$ \\
Gender & \\
Male & $15(65.2)$ \\
Female & $8(34.8)$ \\
Clinical diagnosis & \\
Cataracts & $7(30.4)$ \\
Dry eyes & $6(26.0)$ \\
Blepharitis & $5(22.7)$ \\
Vitreous syneresis & $3(13.0)$ \\
Other & $2(8.7)$ \\
\hline DD: standard deviation &
\end{tabular}

Table II. Comparison of intraocular pressure in the left eye based on pillow type and position.

\begin{tabular}{lcccc}
\hline Position & \multicolumn{3}{c}{ Mean IOP \pm SD (mmHg) } & p-value \\
\cline { 2 - 4 } & Soft pillow & Hard pillow & Difference & \\
\hline $\begin{array}{l}\text { Supine } \\
(n=23)\end{array}$ & $14.48 \pm 3.09$ & $13.65 \pm 3.58$ & $0.83 \pm 2.95$ & 0.193 \\
$\begin{array}{l}\text { Lateral } \\
(n=23)\end{array}$ & $17.48 \pm 3.18$ & $16.65 \pm 3.54$ & $0.83 \pm 2.62$ & 0.145 \\
\hline
\end{tabular}

IOP: intraocular pressure; SD: standard deviation

Table III. Comparison of intraocular pressure in the left eye based on position.

\begin{tabular}{lcccc}
\hline Pillow type & \multicolumn{2}{c}{ Mean IOP \pm SD (mmHg) } & \multirow{2}{*}{ p-value } \\
\cline { 2 - 4 } & Supine & Lateral & Difference & \\
\hline $\begin{array}{l}\text { Soft } \\
(n=23)\end{array}$ & $14.48 \pm 3.09$ & $17.48 \pm 3.18$ & $-3.00 \pm 3.36$ & $<0.001$ \\
Hard & & & & \\
$(n=23)$ & $13.65 \pm 3.58$ & $16.65 \pm 3.54$ & $-3.00 \pm 2.54$ & $<0.001$ \\
\hline
\end{tabular}

IOP: intraocular pressure; SD: standard deviation

$40 \pm 11 \mathrm{mmHg}$ and a statistically significant agreement of $78 \%$ between the eye with a greater cup-disc ratio and the side usually slept on. The authors also believed that during sleep, the weight of the head was transmitted to the globe in eyes that were more consistently opposed to bedding surfaces. ${ }^{(6)}$ The results of our study support the findings of Korenfeld and Dueker. In glaucoma patients with progressive glaucomatous optic neuropathy despite apparent adequate therapy, we believe that it is important to consider sleeping posture as a cause. It is also possible that transient elevations of IOP in the dependent eye when in the lateral decubitus position may, to some extent, account for some patients with normal tension glaucoma. These patients, who show normal pressure recordings in the clinic during waking hours, are known to develop raised IOP while sleeping. Therefore, we propose that sleep studies measuring IOP over time might be needed for patients with normal tension glaucoma.

The authors are aware that this study is not without its shortcomings. First, IOP was measured within minutes of posture adjustment in our study. We know from the literature that autoregulation of ocular blood flow, and thereby the IOP, occurs with changes in posture. ${ }^{(8)}$ Thus, a more reliable approach might have been to wait for about five minutes after the initiation of posture before checking IOP, in order to allow for such autoregulation to take effect. Nevertheless, it is interesting to 
note that our study, which measured IOP within one minute of the initiation of posture, found a significant elevation of IOP in the lateral decubitus position, similar to the study by Korenfeld and Dueker, which measured IOP during a five-minute simulated sleep session. ${ }^{(6)}$ Plots of IOP changes over time in the supine and lateral decubitus postures would also have been useful.

Second, while taking IOP measurements, we ensured that there was some contact between the pillow and patients' eyelids over the temporal globe. This was done to more accurately simulate real-life scenarios where compression of the eyelid on the globe is deemed inevitable. As a consequence, it might be argued that our findings of higher IOP in the lateral decubitus position were biased, as raised IOP in this position may have been partly contributed to by direct mechanical compression of the eye by the eyelid and orbital soft tissues, rather than solely being the result of a change in sleeping position. The authors agree that measuring IOPs with the patient lying laterally with no direct contact between the pillow and the eye would have helped to eliminate such bias. It would also have been useful if the differences in IOP measurements during upgaze (Bell's phenomenon during sleep) and downgaze - due to the effect of compression of the globe by the orbital soft tissues - had been determined.

Third, our study was limited by a small sample size. Although the difference in IOPs measured when lying laterally and supine was statistically significant in our patients, a larger sample size would have been more representative. Even so, the authors are of the opinion that the main aim of this pilot study, which was to observe if different sleeping postures in normal controls were associated with a significant change in IOP, was satisfied. Our results could be used as the basis for more elaborate future studies. Fourth, the time of IOP measurement was not standardised among our patients. IOP was measured between 8 am and $6 \mathrm{pm}$, depending on the time the patients were recruited from the clinic. While IOP is known to show diurnal fluctuations, the authors contend that such variations would not have affected the readings in our study, as IOP measurements in the supine and lateral positions were taken minutes apart for all patients. Also, the authors were only interested in observing changes in IOP due to different positions. Finally, IOP measurements were only performed for the left eye, and it might be interesting to explore whether our findings are reproducible with the fellow eye. The authors, however, believe that IOP trends would be similar in both eyes.

Future studies aimed at exploring whether the elevation in IOP due to different positions is more significant in patients with angle-closure glaucoma as compared to those with open-angle glaucoma would be useful. As eyes that are susceptible to angleclosure are generally smaller and have shorter axial lengths, it is possible that direct mechanical compression of such eyes would bring about a greater rise in IOP when compared to larger eyes.

In conclusion, we found that the lateral decubitus position, which is commonly adopted during sleep, was associated with a significant increase in the IOP of the dependent eye. While changes in hydrostatic responses in the episcleral venous pressure and the distribution of body fluid are possible causes for the rise seen in IOP in the supine position, ${ }^{(8)}$ the authors believe that mechanical compression is a key cause of IOP increase when lying in the lateral decubitus position. This association may be further explored in larger case-control studies. Our findings in normal patients indicate that sleep posture has an important role to play in glaucoma patients and that nocturnal elevation of IOP may be a cause of glaucoma progression in this cohort.

\section{REFERENCES}

1. Musch DC, Gillespie BW, Niziol LM, et al. Factors associated with intraocular pressure before and during 9 Years of treatment in the Collaborative Initial Glaucoma Treatment Study. Ophthalmology 2008; 115:927-33.

2. Prata TS, De Moraes CG, Kanadani FN, Ritch R, Paranhos A Jr. Postureinduced intraocular pressure changes: considerations regarding body position in glaucoma patients. Surv Ophthalmol 2010; 55:445-53.

3. Hara T, Hara T, Tsuru T. Increase of peak intraocular pressure during sleep in reproduced diurnal changes by posture. Arch Ophthalmol 2006; 124:165-8.

4. Kiuchi T, Motoyama Y, Oshika T. Relationship of progression of visual field damage to postural changes in intraocular pressure in patients with normal-tension glaucoma. Ophthalmology 2006; 113:2150-5.

5. Mehdizadeh M. Sleep position and eye pressure. Ophthalmology 2007; 114:2632; author reply 2632.

6. Korenfeld MS, Dueker DK. Occult intraocular pressure elevation and optic cup asymmetry, sleep posture may be a risk factor. Invest Ophthalmol Vis Sci 1993; 34(suppl):994.

7. Liu JH, Kripke DF, Hoffman RE, et al. Nocturnal elevation of intraocular pressure in young adults. Invest Ophthalmol Vis Sci 1998; 39:2707-12.

8. Evans D.W, Harris A, Garrett M, Chung HS, Kagemann L. Glaucoma patients demonstrate faulty autoregulation of ocular blood flow during posture change. Br J Ophthalmol 1999; 83:809-13. 